\title{
THE VERTICAL AGE DISTRIBUTION OF STELLAR DISK POPULATIONS IN EDGE-ON GALAXIES
}

\author{
A. JUST, B. FUCHS AND R. WIELEN \\ Astronomisches Rechen-Institut \\ Mönchhofstr. 12-14, D-69120 Heidelberg, Germany
}

The scale height of the disk stars in our Galaxy increases continously with age, which is also seen in the age-velocity dispersion relation of the solar vicinity. In external galaxies a similar evolution of stellar disks can be deduced from the colour distribution of the light in edge-on galaxies. Using physical disk models and stellar population synthesis we compute the luminosity and colour profiles in optical and infrared bands. For diffusion coefficient and SFR constant in time the vertical density profile is exponential. The colour profiles are mainly affected by dust extinction, and the age and metallicity distribution of the stars. The comparison of a basic model with the observed profiles of IC 2531 shows, that these influences can be disentangled by analyzing the systmatic deviations in the colour profiles.
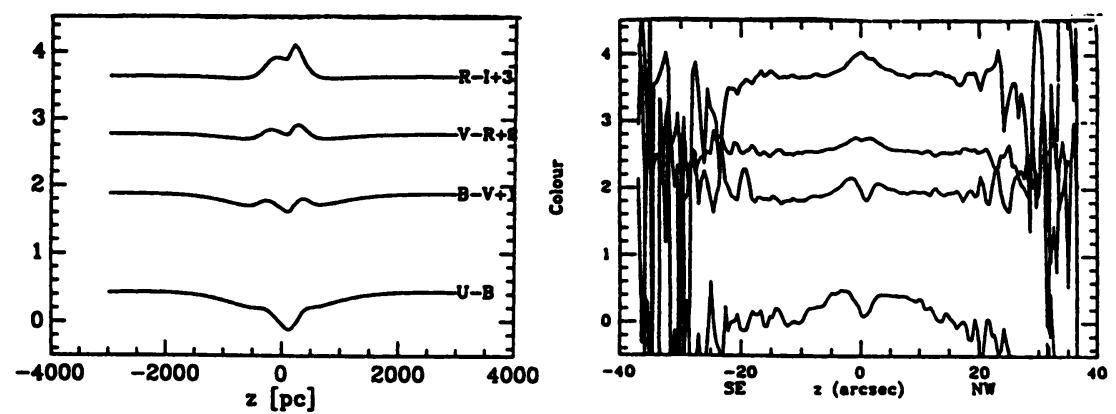

Figure 1. Computed colour profiles of IC 2531; observations from Wainscoat et al. 1989.

\section{References}

Just, A., Fuchs, B., Radons, G., Wielen, R. (1993) The Effect of Dynamical Evolution of the Disk on the Colour Distribution of Edge-on Galaxies Panchromatic View of Galaxies, Editions Frontières, pp. 292-295 